eJKI | Vol. 9, No. 2, September 2020

Jurnal Kesehatan Islam

Publikasi oleh Fakultas Kedokteran, Universitas Islam Malang

Pages: 60-82

Email: jkesislam@unisma.ac.id

Home Page : http://riset.unisma.ac.id/index.php/jki

\title{
MANAGEMENT OF CARDIOGENIC SHOCK IN PEDIATRIC PATIENTS
}

\author{
Dyahris Koentartiwi, Naura A Candini, Yunita L Indrianto, Renny Suwarniaty
}

Department Pediatrics

RSUD Dr Saiful Anwar - Faculty of Medicine Universitas Brawijaya

Malang

\begin{abstract} prevent complication and mortality.

Keywords : cardiogenic shock, children, diagnosis, treatment

Correspondence author:

dr. Dyahris Koentartiwi, Sp.A (K)

RSUD Dr Saiful Anwar - Faculty of Medicine Universitas Brawijaya

Email : dyahris08.fk@ub.ac.id
\end{abstract}

Cardiogenic shock is an acute state of end-organ hypoperfusion following cardiac failure. Usually many children have good compensation if they suffered from cardiogenic shock and sometimes delay diagnosis leads to unfavorable outcome. Comprehensive approach in treatment following investigation about the cause of cardiogenic shock is very important and proper management will

Shock is an acute process in which the body is unable to deliver adequate oxygen to meet the metabolic demands of vital organs and tissues. The lack of oxygen at tissue level will cause a shift to anaerobic metabolism which is less efficient. As shock progresses, it can lead to adverse responses such as on vascular system, inflammatory reactions, cellulary and metabolic consequences, endocrine system, and systemic results. Subsequently, it cause worsen physiologic instability. Cardiogenic shock is often seen in children with congenital heart disease and cardiomyopathies (Kliegman et al., 2020).
Cardiogenic shock is an acute state of end-organ hypoperfusion following cardiac failure. There are many causes of it such as primary pump failure, and often accompanied by inadequate preload or afterload (Chiwane et al., 2018). Cardiogenic shock is often characterized by low cardiac output which can result in an inadequate tissue perfusion (Kliegman et al., 2020).

\section{Epidemiology}

Shock occurs in approximately $2 \%$ of all hospitalized infants, children, and adults in developed countries, and the 
mortality rate varies substantially depending on the etiology and clinical circumstances. The most common cause of death is rarely due to the acute hypotensive phase of shock, but rather as a consequence of associated complications and multi-organ dysfunction (Kliegman et al., 2020).

Cardiogenic shock represents 5 $13 \%$ of diagnosed cases of shock in pediatric emergencies. It is the most advanced and most serious stage of heart failure. In hospitalized children, cardiogenic shock is lethal in $5-10 \%$ of cases. The mortality rate is similar to that observed in adults (Brissaud et al., 2016). Generally, mortality rates in cardiogenic shock remains as high as $35-$ 50\% (Kataja and Harjola, 2017).

\section{Etiology}

Cardiogenic shock can be caused by various etiologies that also varies between ages. In neonates, cardiogenic shock can be caused by birth asphyxia and sepsis, as well as congenital heart disease. In infants and older children, cardiogenic shock can be caused by infection diseases and other causes of cardiomyopathies (Chiwane et al., 2018). Causes of cardiogenic shock according to age of occurrence can be seen in Table 1 .

Table 1. Causes of cardiogenic shock due to age-related (Chiwane et al., 2018).

\begin{tabular}{|c|c|c|}
\hline Age & Causes of cardiogenic shock & \\
\hline Day 1 of life & $\begin{array}{l}\text { - } \text { Birth asphyxia } \\
\text { - Congenital heart disease (TAPVR } \\
\text { with obstructions, TGA with IVS } \\
\text { with restricted atrial septum, HLHS } \\
\text { with restricted atrial septum) }\end{array}$ & $\begin{array}{l}\text { - Sepsis } \\
\text { - Fetal/neonatal myocarditis } \\
\text { - Hypocalcemia } \\
\text { - Hypoglycemia } \\
\text { - Brady/tachyarrythmia }\end{array}$ \\
\hline First week of live & $\begin{array}{l}\text { - } \text { Ductal dependent systemic } \\
\text { circulation (HLHS, critical aortic } \\
\text { stenosis, interrupted aortic arch) } \\
\text { - Sepsis }\end{array}$ & $\begin{array}{l}\text { - Congenital adrenal } \\
\text { insufficiency and inborn errors } \\
\text { of metabolism }\end{array}$ \\
\hline $2-6$ weeks & $\begin{array}{l}\text { - VSD } \\
\text { - Complete AV canal defects } \\
\text { - Aortopulmonary window } \\
\text { - Truncus arteriosus } \\
\text { - Unobstructed TAPVR } \\
\text { - Persistent PDA }\end{array}$ & $\begin{array}{l}\text { - ALCAPA } \\
\text { - Coarctation of aorta } \\
\text { - Pompe's disease } \\
\text { - Sepsis } \\
\text { - Myocarditis/cardiomyopathy }\end{array}$ \\
\hline 6 weeks to 1 year & $\begin{array}{l}\text { - } \text { Coarctation of aorta } \\
\text { - Aortic stenosis } \\
\text { - Sepsis } \\
\text { - Kawasaki disease }\end{array}$ & 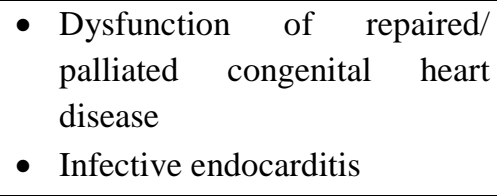 \\
\hline $\begin{array}{l}\text { Older children and } \\
\text { adolescents }\end{array}$ & $\begin{array}{l}\text { - Arrythmias } \\
\text { - Acute rheumatic fever } \\
\text { - Infective endocarditis } \\
\text { - Acute aortic insufficiency } \\
\text { - Cardiomyopathy }\end{array}$ &  \\
\hline
\end{tabular}


- Drug ingestions: calcium channel

and beta-blockers

- Hypertensive emergencies

N.B. TAPVR: total anomalous pulmonary venous return, TGA: transposition of great arteries, IVS: intact ventricular septum, HLHS: hypoplastic left heart syndrome, AV: atrioventricular, PDA: patent ductus arteriosus, ALCAPA: anomalous left coronary artery from the pulmonary artery. Arrythmias, myocarditis, cardiomyopathies, sepsis, and electrolyte disturbances can cause cardiogenic shock at all ages.

According to the underlying pathologic mechanisms, cardiogenic shock is found as a result of the dysfunction of systolic and diastolic phases, increased afterload, structural abnormality, and arrythmia. Systolic dysfunction is caused by decreased contractility due to ischemia, valvular diseases, acidosis, and the use of drugs such as beta-blockers, calcium channel blocker, and anti-arrythmia drugs. Diastolic dysfunction is usually caused by myocardial stiffness due to ischemia, hypertrophy, cardiomyopathies, and external compressions such as pericardial tamponade. Increased afterload may be caused by a multitude of things such as aortic stenosis, cardiomyopathies, coarctation of aorta, and pulmonary embolism. Structural abnormality may be caused due to congenital abnormality such as mitral stenosis and mitral aortic regurgitation, or due to secondary causes such as endocarditis and thrombus (Kliegman et al., 2020; Chiwane et al., 2018).

\section{Pathophysiology}

Cardiogenic shock is potentially caused by severe cardiac dysfunction at before or after cardiac surgery, septicemia, severe burns, anaphylaxis, cardiomyopathy, myocarditis, myocardial infarction and acute central nervous system disorders (Kliegman et al., 2020).

Cardiogenic shock, a circulatory shock is a product of hypoperfusion due to oxygen imbalance. Oxygen consumption is the amount of oxygen used by the tissues. It is the difference between the oxygen delivered by the arterial system and the amount of oxygen returned to the heart by the venous system. In healthy individuals, oxygen consumption is equal to oxygen demand. Oxygen delivery is the product of cardiac output multiplied by arterial oxygen content. Cardiac output itself, is the product of heart rate multiplied by stroke volume (Chiwane et al., 2020).

Cardiac output can be reduced by decreased preload (e.g., hypovolemia), decreased contractility (e.g., myocarditis), increased afterload (e.g., coarctation of aorta), and atrioventricular asynchrony (e.g., total heart block). Meanwhile, arterial oxygen content can be decreased by anemia, abnormal hemoglobin concentrations (carboxyhemoglobin, methemoglobin), shifts in oxygen hemoglobin dissociation curve, and acute hypoxemic respiratory failure. All of the aforementioned conditions may lead to an imbalance between oxygen delivery and 
oxygen demand, which then may lead to shock (Chiwane et al., 2018).

In the case of cardiogenic shock in the pediatric population, the oxygen imbalance can be caused by a multitude of things. In ventricular septal defects, the leftto-right shunting can cause a decrease of preload in the left ventricle which can reduce cardiac output. In obstructive lesions such as the coarctation of aorta, the obstruction will cause difficulty for the heart to generate sufficient force to overcome said obstruction which can compromise preload and later on, cardiac output. In the cases of rheumatic heart diseases, inflammation may cause cardiomyopathies that can impede contractility, thus reducing stroke volume. In arrythmias, stroke volume may be affected due to the irregularity of stroke volume. Aside from cardiac causes, reduced cardiac output can also be caused due to pneumothorax and large pulmonary embolus, as well as electrolyte abnormalities such as hyperkalemia or hypocalcemia which may affect heart rate (Kliegman et al., 2020).

Generally, shock follows three distinct stages regardless of the classification and underlying diseases, which are compensated shock, uncompensated shock, and irreversible shock (Stephenson, 2020).

In the first stage of compensation, a series of physiological changes occur to ensure that the core essential organs (brain, heart, lungs) are prioritized in terms of oxygenated blood supply. Peripheral vessels then constrict to minimize blood flow to the extremities and the heart increases the rate of blood flow. This mechanism increases systemic vascular resistance (SVR), in which blood restriction to the extremities will improve venous return to the heart, thus increasing the volume of blood ejected in each contraction. During this stage, a child's extremities may feel cold and clammy to the touch as a result of peripheral constriction. This vasoconstriction and poor perfusion can lead to prolonged capillary refill time (CRT) (Stephenson, 2020).

As opposed to adult, children can actually maintain an adequate blood pressure through compensation as compensatory responses maintain homeostasis. Due to this, blood pressure monitoring may not be helpful in recognizing shock in its early compensated form. Due to this, measuring the heart rate may be more reliable to detect shock alongside with signs of peripheral constrictions such as cold and clammy extremities as well as prolonged CRT (Stephenson, 2020).

Once peripheral vasoconstriction and increased cardiac effort failed to compensate for a reduced circulation, shock progresses into an uncompensated state which leads to inadequate tissue perfusion. When tissues are deprived of oxygen, they have to shift their metabolism into an anaerobic one which can result in the increased of lactic acid, a by-product of anaerobic metabolism. This could lead to acidosis and this can be used as a marker of hypoperfusion. When hypoperfusion persists, 
it can lead to reduce in blood flow and impairment of organ function. Some changes can be observed during this stage such as reduced urine output, increased respiratory effort, reduced consciousness, and reduced blood pressure (Stephenson, 2020).

If the efforts to correct underlying diseases and restore an effective circulation fails, patient may fall into a state of irreversible shock. During this stage, tissues recovery is no longer possible and tissues continue to die. Despite continuous efforts to resuscitate and restore circulation, this stage is often irreversible and fatal (Stephenson, 2020).

\section{Diagnosis}

Cardiogenic shock is an emergency; thus, it is important to recognize and treat it while still on its early stage to prevent clinical deterioration and organ damages. The diagnosis of cardiogenic shock is based on a high clinical index of suspicion. Usually, a thorough history and physical examination, close attention to vital signs and response to therapies, and frequent clinical assessments will help make the diagnosis (Chiwane et al., 2018).

More often than not, pediatric patients will display a wide range of symptoms and signs in response to the interaction of cardiac, pulmonary, and even gastrointestinal systems as a result of the direct underlying diseases as well as compromised circulation (Migally and McBride, 2018).

The symptoms and signs, as well as the underlying mechanism can be seen in the following table:

Table 2. Symptoms of cardiogenic shock in children (Chiwane et al., 2018)

\begin{tabular}{l|l}
\hline Symptom & Pathophysiology \\
\hline Feeding difficulty & $\begin{array}{l}\text { - Fatigue from low stroke volume } \\
\text { - Pulmonary congestion from increased left ventricular end-diastolic } \\
\text { pressure }\end{array}$ \\
\hline Failure to thrive & $\begin{array}{l}\text { - Poor calorie intake from feeding difficulty } \\
\text { - Increased myocardial and respiratory muscle caloric demand }\end{array}$ \\
\hline Irritability or lethargy & $\begin{array}{l}\text { - Decreased oxygen delivery to the brain } \\
\text { Dyspnea }\end{array}$ \\
\hline Palpitations & - Myocardial ischemia \\
\hline Sweating & - Tachycardia, bradycardia, or arrythmias \\
\hline Abdominal pain and & - Increased sympathetic activity \\
vomiting & - Congestive hepatomegaly \\
\hline
\end{tabular}

Table 3. Signs of cardiogenic shock in children (Chiwane et al., 2018)

\begin{tabular}{l|l}
\hline Sign & Pathophysiology \\
\hline Cold extremities, weak distal & - Vasoconstriction \\
pulses, and prolonged CRT & - Tissue hypoperfusion \\
\hline Disproportionate tachycardia & - Sympathetic overactivity \\
& - Rate-dependent cardiac output \\
\hline
\end{tabular}




\begin{tabular}{l|l}
\hline Tachypnea & $\begin{array}{l}\text { - Interstitial congestion due to lung tissue J receptor } \\
\text { stimulation in response to increased pulmonary venous } \\
\text { pressure }\end{array}$ \\
\hline Narrow pulse pressure & $\begin{array}{l}\text { Systemic vasoconstriction } \\
\text { - Decreased stroke volume }\end{array}$ \\
\hline Crepitations & $\begin{array}{l}\text { Alveolar edema due to increased pulmonary venous } \\
\text { pressure }\end{array}$ \\
\hline $\begin{array}{l}\text { Dependent edema } \\
\text { hepatomegaly }\end{array}$ & and \\
\hline Gallop rhythm & $\begin{array}{l}\text { Passive venous congestion due to elevated right atrial } \\
\text { pressure }\end{array}$ \\
\hline & - S3: ventricular dilatation due to volume overload \\
& S4: ventricular hypertrophy due to pressure overload \\
\hline
\end{tabular}

Signs and symptoms between each classification of shock may overlap, and the clinical presentation usually depends on the underlying etiology. If unrecognized and untreated, all forms of shock can progress to irreversible organ injury and death. Shock may initially manifest as only tachycardia, with or without tachypnea, which later progress to decreased urine output, poor peripheral perfusion, respiratory failure, alteration of mental status, and low blood pressure. There is a misconception in which shock occurs with low blood pressure.

Table 4. Normal heart rate and hypotensive systolic blood pressure in pediatric patients (Mendelson, 2018)

\begin{tabular}{|l|l|l|}
\hline Age & Heart rate $(\mathrm{bpm})$ & Hypotensive $\mathrm{SBP}(\mathrm{mmHg})$ \\
\hline$<1$ mo. & $110-180$ & $<0$ \\
\hline $1-12$ mo. & $100-170$ & $<70$ \\
\hline $1-2$ y.o. & $85-150$ & $<70+(2 \mathrm{x}$ age in years $)$ \\
\hline $3-5$ y.o. & $70-140$ & $<70+(2 \mathrm{x}$ age in years $)$ \\
\hline $6-10$ y.o. & $60-110$ & $<70+(2 \mathrm{x}$ age in years $)$ \\
\hline$>10$ y.o. & $50-100$ & $<90$ \\
\hline
\end{tabular}

As previously mentioned, cardiogenic shock can be caused by various conditions and the most common etiology for cardiogenic shock in children differs to those in adults. In adults, cardiogenic shock is often
Children differs from adult in which low blood pressure is often found as late sign due to their ability to compensate through increasing stroke volume by elevating the heart rate. Thus, tachycardia may be a more significant finding in diagnosing shock (Kliegman et al., 2020).

Pediatric heart rate ranges and systolic blood pressure level to determine hypotension can be seen in the following table: 
prominently, cardiac failure due to congenital heart diseases. Despite the difference causes, cardiogenic shock and cardiac failure hemodynamic status in children can be categorized in the same way as it does in adult according to the presence or absence of two traits: venous congestion and hypoperfusion. This is known as the warm, cold, wet, and dry concept, and is beneficial in choosing the right treatment later on (Mendelson, 2018).
The presence of venous congestion is owed to the increased filling pressure, which is considered "wet", while the absence is considered "dry". Meanwhile, the presence of hypoperfusion is owed to the decreased cardiac output and myocardial contractility, which is considered "cold", while normal perfusion is considered "warm". The illustration and description of the aforementioned concept can be seen in the following diagram:



Figure 1. Illustration of patient's hemodynamic status in cardiogenic shock (Mendelson, 2018)

Even though diagnosis of cardiogenic shock can simply be made from clinical signs and symptoms, certain tests may be required, mainly to define the underlying cause of the shock itself, to assess the myocardium functional status and other comorbid features, as well as to monitor therapeutic response to the treatments given (Kliegman, et al., 2020).

Several studies can be used to determine the underlying causes of shock. Imaging studies are the mainstay to diagnose congenital abnormalities that may results in shock. Chest radiography is often used as it is relatively cheap and widely available. Chest 
radiography can help excluding other causes of shock and chest pain, as well as assessing pulmonary vasculature and cardiomegaly. Electrocardiography (ECG) is commonly performed to identify rhythm disturbance and structural diseases such as ALCAPA (Kliegman et al., 2020).

Ultrasound based studies is also a useful tool for it is relatively easy and quick to operate, and it has no radiation side-effects. Focused assessment with sonography for trauma (FAST) is used routinely both in children and adults alike to identify hemoperitoneum, hemopericardium, and hemothorax, as well as pneumothorax in extended FAST (e-FAST) (Mendelson, 2018)

Ultrasound guided echocardiography (echo) is often used to diagnose anatomic abnormalities, ascertain functional status, and to follow-up patient's condition after therapy (Kliegman et al., 2020). A parameter that can be assessed using echo is myocardial performance index (MPI). MPI is a simple, reproducible, and noninvasive measure to assess the systolic and diastolic function in comparison of isovolumetric contraction (ICT) and relaxation time (IRT) (Hayabuchi et al., 2019). MPI can be measured through the following equation:

$$
\text { MPI: } \frac{(I C T+I R T)}{\text { VET }}
$$

When systolic function worsens, ICT lengthens, the VET shortens, and subsequently the MPI increased. Meanwhile, when diastolic function worsens, MPI will also increase but due to the lengthening of IRT (Hayabuchi et al., 2019).

Laboratory studies are also commonly performed to discover hematologic abnormalities and electrolyte disturbances. Hematologic abnormalities may include thrombocytopenia, prolonged prothrombin and partial thromboplastin times, reduced serum fibrinogen level, elevation of fibrin split products, and anemia. Elevated neutrophil counts and increased immature forms (i.e., bands, myelocytes, promyelocytes), vacuolation of neutrophils, toxic granulations, and Döhle bodies can be seen with infection (Kliegman et al., 2020).

\section{Hyperglycemia or hypoglycemia} may appear as a stress response. Electrolyte abnormalities such as hypocalcemia, hypoalbuminemia, and metabolic acidosis can also be present (Kliegman et al., 2020).

Central venous pressure measurement can be performed to approximates right arterial pressure to assess preloading condition. Mixed venous oxygen saturation $\left(\mathrm{SvO}_{2}\right)$ is considered as the balance between oxygen demand and delivery, and has been used as a determinant for tissue hypoxia (Singh et al., 2018).

\section{Management and Treatment}

Children who come to the
emergency department with signs or
symptoms of shock need a quick evaluation
and stabilization without any delay. Initial
treatment is focused on restoring enough


oxygen delivery to peripheral tissue. All patient that come with cardiogenic shock should be placed and treated in pediatric intensive care unit (PICU). Assessment is consisted of stabilization of airway, breathing, and circulation, disability, exposure and continued by assessment of clinical history, physical examination, and laboratory examination. Early goal directed therapy (EGDT) is focused in maintaining and restoring airway patency, oxygenation, ventilation, and circulation (perfusion, normal blood pressure, and normal heart rate based on age) on the first hour of shock onset. Oxygen should be administrated for any kind of shock despite the etiologies. Monitor and evaluate oxygen saturation and give intravenous (IV) access immediately. Fluid resuscitation is needed immediately to correct hypovolemia and hypotension situation with precaution of pulmonary edema (Pasman, 2019; Ren, 2019).
Basic treatment for any kind of shock is to optimize oxygen delivery so that it can meet the oxygen demand. The treatment is focused in increasing oxygen delivery and decreasing oxygen demand. Evaluate and correct hypoglycemia and hypocalcemia $(<1.1 \mathrm{mmol} / \mathrm{L})$. Therapeutic goals for management of pediatric shock should include the following points (Pasman, 2019):

1. Normal mental status

2. Normal blood pressure in accordance with age

3. Normal or threshold heart rate in accordance with age

4. Normal and equal both central and peripheral pulse

5. Warm extremities and capillary refill 2 second or less

6. Normal urine output $(>1 \mathrm{~mL} / \mathrm{kg} / \mathrm{h})$

7. Normal serum glucose levels

8. Normal serum calcium level

9. Decrease in lactate serum level 



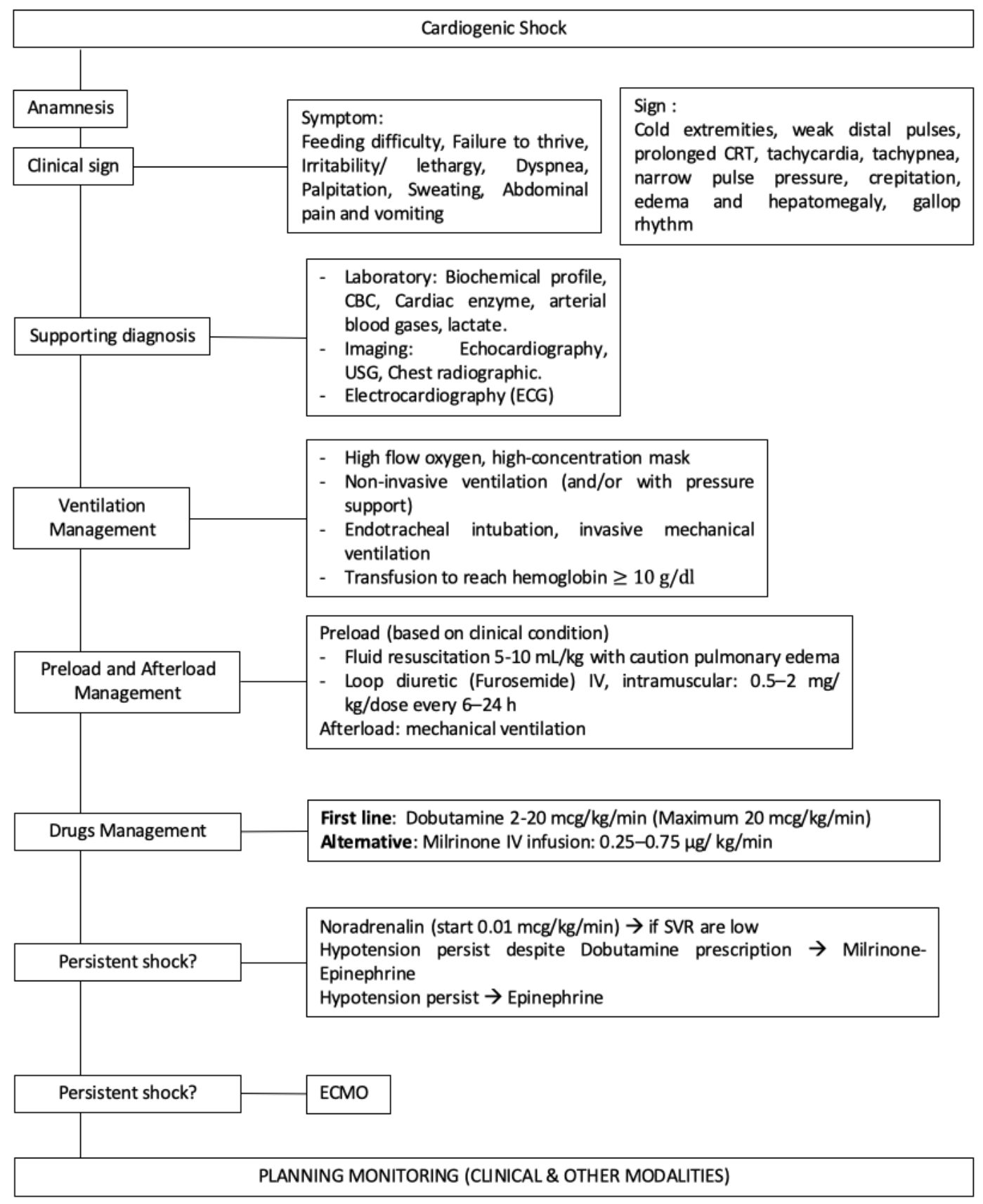

Figure 2. Algorithm of Pediatric Cardiogenic Shock (Brissaud et al., 2016; Pasman, 2019).

\section{Optimizing preload using diuretics and}

\section{fluid administration}

Fluid resuscitation should be given in any kind of shock. Isotonic crystalloid infusion can be administered for $20 \mathrm{~mL} / \mathrm{kg}$ for 20 minutes. This crystalloid infusion can be given through intravenous (IV) or intraosseous (IO). But an exception given for children with cardiogenic shock. In neonates or children with cardiogenic shock, crystalloid fluid $5-10 \mathrm{~mL} / \mathrm{kg}$ boluses can be administrated. For children suspected with 
right ventricular dysfunction or hypertrophy need higher preload to correct contractility. For this patient fluid challenge can give more advantage, slowly administer small dose of aliquots $5 \mathrm{~mL} / \mathrm{kg}$. Reassess frequently to evaluate any signs of volume overload (hepatomegaly, S3 gallop, or rales/crackles), increased jugular venous pressure, and poor perfusion (Ren, 2019).

Meanwhile in children with left ventricular dysfunction diuretic can be administered to decrease preload. Diuretics works by decreasing plasma volume and peripheral edema causing decreased cardiac output, and blood pressure with increasing peripheral vascular resistance as compensation mechanism. Diuretic mainly used to treat systemic and pulmonary vascular congestion. Diuretic agent is classified into four subgroups (Ren, 2019):

\section{A. Loop diuretics}

Most common used loop diuretic is furosemide. Furosemide can be used to treat high preload in cardiogenic shock. Furosemide work by increasing the excretion of water by inhibiting reabsorption of sodium and chloride in the ascending loop of Henle and distal renal tubule. Except furosemide loop diuretic example is bumetanide dan Ethacrynic acid. Furosemide is preferred among another diuretic because its rapid onset of action and long duration for almost 3 hours. Furosemide can be given as a bolus or as infusion. Adverse effect of this medication includes hypokalemia, metabolic alkalosis, hypocalcemia, hyponatremia, and hyperuricemia (Ren, 2019).

B. Potassium-sparing diuretics

Spironolactone

(main mineralocorticoid (aldosterone) receptor antagonist) is the most common potassiumsparing diuretic used. Spironolactone use is limited because its nature as weak diuretic and it's not preferred to be used as a single medication. Spironolactone can be used to treat edema in pediatric with congestive heart failure caused by excessive aldosterone produced. Spironolactone competes with aldosterone for receptor sites in distal renal tubules, making water excreted while potassium and hydrogen ions retained. Potassium-sparing diuretic is usually given orally few days after the patient has been stabilized (Satou, 2019).

C. Thiazide diuretics

Thiazide can be differentiated into two classifications, hydrochlorothiazide and chlorothiazide. Compared to furosemide, thiazide agents have slower effect. Thiazide commonly prescribed for children with congestive heart failure, hydrochlorothiazide inhibits sodium reabsorption in distal tubules. Sodium reabsorption causes increased sodium, water, potassium and hydrogen ions excretion (Satou, 2019).

D. Thiazide-like diuretics

Commonly used thiazide-like diuretics is metolazone and there is other agent of thiazide-like diuretics such as indapamide and chlorthalidone. Metolazone 
mechanism is similar to thiazide diuretic by inhibiting sodium resorption at cortical diluting site and proximal convoluted tubule (Ren, 2019).

\section{Improving myocardial contractility}

Vasoactive agent should be given to patient with persistent shock after administration of initial fluid resuscitation. Initial treatment with inotropic agents through peripheral vascular access is recommended. Specific inotropic administration based on hemodynamics, cardiac output, systemic vascular resistance (SVR) (Pasman, 2019).

Cardiac contractility can be improved with inotropic medication. Inotropic drug includes catecholamines, phosphodiesterase inhibitor (PDE Inhibitor), and calcium sensitizers. Almost all inotropic agents increase myocardial oxygen consumption and has potential to induce arrhythmia. Receptor response on organs and tissue should be considered when vasoactive agent is indicated. Vasoactive agents cause cardiac output and systemic vascular resistance will increase by inducing contractility, heart rate, and peripheral vasoconstriction (Chiwane et al., 2018). Based on general classification of inotropic and vasoactive agent (vasopressors or vasodilators) characteristic, inotropic and vasoactive agents can be classified into four subclasses (Dabbagh et al., 2017):

E. Pure vasopressor (pure vasoconstrictor)

1. Phenylephrine
Phenylephrine stimulates in alpha-1 agonist receptor which causes vasoconstriction in peripheral artery helping the raise of blood pressure. The vasoconstriction effect may cause bradycardia reflex though this condition is rarely found in pediatric patient. Phenylephrine most important indication is low SVR in CHD patient (Tetralogy of Fallot (TOF), hypertonic cardiomyopathy, etc.). This agent is also given in patient with partial obstruction in systemic to pulmonary shunt or pulmonary shunt in single ventricle patient with pulmonary stenosis to improve oxygenation. Phenylephrine dosage in pediatric patients: Bolus $0.5-5 \mathrm{mcg} / \mathrm{kg}$ or higher and Infusion 0.02-0.3 mcg/ kg/min (Dabbagh et al., 2017).

Phenylephrine induces cardiac output and cause angina exacerbation, heart failure, and pulmonary hypertension. Side effect can be found in phenylephrine administration including vasoconstriction of peripheral vascular that can be severe and disturb blood flow in vital organ, nausea, vomiting, headache, and nervousness. Precaution is needed in administrating phenylephrine because there's high risk of extravasation into skin and subcutis. Ischemia, even necrosis and tissue lost can happen as result of extravasation. Absolute contraindication in phenylephrine is patient with hypersensitivity to the agent. Phenylephrine has component of sodium metabisulfite that can cause allergic-type reactions, including anaphylactic in some people (Richard et al., 2019). 
2. Vasopressin

Vasopressin has three different receptors (V1a receptor, V1b receptor (or V3 receptor), dan V2 receptor). Vasopressin or ADH (Antidiuretic Hormone) exogen can be used after hemodynamic stable and commonly given in patient with vasodilatory shock. In pediatric patient with septic shock, vasopressin could be administered after agent like norepinephrine given before. Vasopressin exerts intense vasoconstriction and antidiuretic effects. Vasopressin works by stimulating vascular smooth muscle contraction through $\mathrm{V} 1 \mathrm{a}$ receptor and on the other side from V2 receptor, vasopressin plays an important role in regulating water and sodium homeostasis. V1b receptor or V3 receptor is restricted to central nervous system (Bankir et al., 2017).

Vasopressin is indicated in pediatric patient with diabetes insipidus, polyuria, $\mathrm{CPR}$, diagnostic procedure, and vasodilatory shock. In excessive alpha-adrenergic blockade, vasopressin is proved to be useful in treating low systemic vascular resistance. Commonly used dosage or administration is 0.2-2 milliunits $/ \mathrm{kg} / \mathrm{min}$ infusion, which can be given by titration until desired effect achieved but Vasopressin only can be given in short term period. It is reported that administration of vasopressin for 4-8 milliunits $/ \mathrm{kg} / \mathrm{min}$ can treat vasodilatory shock. Vasopressin usually give no significant adverse effect in low dose; however, the severity of adverse effect can rise together with increasing dosage. Adverse effect that can occur include hypertension and bradycardia due to severe vasospasm, limb ischemia due to vasoconstriction, necrosis due to extravasation, hyponatremia in prolong infusion, and hypersensitivity in susceptible patient (Dabbagh et al., 2017).

F. Inoconstrictors (vasoconstrictor and inotropic activity)

1. Epinephrine

Epinephrine effects in all adrenergic receptors as example alpha-1, alpha-2, beta1 , and beta-2. Epinephrine is recognized as the most potent alpha-adrenergic agonist Epinephrine with lower dose infusion $(<0.1$ $0.2 \mathrm{mcg} / \mathrm{kg} / \mathrm{min}$ ) affect predominantly in beta adrenoceptor and considered as "pure inotropic" dose. Vasoconstrictor trait of epinephrine will increase with the increasing dosage. Epinephrine cause dilatation of smooth muscles in bronchi and iris dilatation, increasing blood glucose due to glycogenolysis, myocardial ischemia, tachyarrhythmia, and lactic acidosis Perfusion of hepatic and splanchnic will decrease and it can induce high hepatic metabolic workload, hypermetabolism, oxygen impairment, glycolysis, and insulin suppression which can lead to lactic acidosis and hyperglycemia condition. Epinephrine is indicated in patient with urgency cardiopulmonary resuscitation and rhythm disturbances, bronchospasm, and anaphylaxis shock. (Dabbagh et al., 2017).

2. Dopamine 
Dopamine is a dose dependent agent which nature is defined by the dosage given. Dopamine induce chronotropic and inotropic effects on myocardium which can induce heart rate and myocardial contractility. Alpha- and beta-adrenergic receptors of Dopamine are weaker compared to Epinephrine and Norepinephrine. Dopamine effect needs 5 min of time to start the effect, on the other side it takes only less than 10 minutes for systemic effect disappeared. Side effect of dopamine is dose dependent. Dopamine is indicated in patient with shock, acute renal failure, hepatorenal syndrome, cardiopulmonary resuscitation, and heart failure. Dopamine effect can be classified based on the dosage (Dabbagh et al., 2017):

A) Low-dose dopamine $(0.5-2 \mathrm{mcg} / \mathrm{kg} / \mathrm{min})$ will induce vasodilation effect

Clinical presentation of low-dose dopamine: Increased glomerular filtration rate (GFR), increased renal blood flow, increased renal excretion of sodium, and increased urine flow

B) Medium-dose dopamine $\mathrm{mcg} / \mathrm{kg} / \mathrm{min}$ ) will stimulate beta-1 receptor (beta-2 receptor is not stimulated by this dose)

Clinical presentation of medium-dose dopamine includes increase myocardial contractility, increase sinoatrial node (SA), increase impulse conduction, increase systolic pressure (diastolic is not affected much).

C) High-dose dopamine $\mathrm{mcg} / \mathrm{kg} / \mathrm{min}$ ) mainly effects alpha receptor
Clinical result of high-dose dopamine administration include vasoconstriction, elevated blood pressure, renal and mesenteric vessel could be affected by increasing the dosage.

D) Very high dopamine dose $\quad(>20$ $\mathrm{mcg} / \mathrm{kg} / \mathrm{min}$ ) could lead to ischemia Limb circulation will be compromised and lead to ischemia condition. Prescription of very high dose of dopamine will give the same side effects as norepinephrine.

Stopping Dopamine administration need to be decreased gradually while maintaining blood volume with IV fluid to prevent hypotension (Dabbagh et al., 2017). Dopamine prescription is usually not recommended due to dopamine potential to cross blood-brain barrier and suppress pituitary hormones. Side effect that commonly occur with administration of Dopamine include tachycardia, angina, palpitation, vasoconstriction, hypotension, dyspnea, nausea and vomiting, and headache (Dabbagh et al., 2017).

3. Norepinephrine

Norepinephrine, similar to epinephrine, stimulates beta-1 and alphaadrenergic receptors, causing increase in myocardial contractility, heart rate, and systemic vasoconstriction. Even though norepinephrine is a potent alpha- 1 receptor, it gives not significant effect on beta-2 receptors (which responsible for vasodilation). It also can cause increase in systemic vascular retention and blood 
pressure with low dose. Cardiac output will be decreased or maintained, and heart rate will decrease due to vagal reflex. Prolong infusion will induce hyperglycemia higher than epinephrine. Norepinephrine is indicated in patient with unresponsive to other vasopressor agent with need of very potent vasoconstrictor such as, shock (unresponsive to dopamine or dobutamine in neonates with septic shock), anaphylactic shock, myocardial infarction, ACLS severe hypotension, and pericardial tamponade. Norepinephrine is given intravenous (IV) infusion $0.02-0.2 \mathrm{mcg} / \mathrm{kg} / \mathrm{min}$, and it is recommended to use smallest dose and in shortest time. In giving norepinephrine, some precautions should be aware of including prolong administration (as it could induce cardiac output decrease, edema, hemorrhage, necrosis of organs due to severe shock or due to shock itself), severe vasoconstriction, increase myocardial oxygen consumption and the work of heart, arrythmias, dizziness, tremor, and headache (Dabbagh et al., 2017).
G. Inodilator (vasodilator and inotropic activity)

\section{Milrinone}

Milrinone is one of phosphodiesterase inhibitor agent. Milrinone works by inhibiting PDE3 which leads accumulation of cyclic adenosine monophosphates (cAMP). It can stimulate and increase cardiac output. cAMP has vasodilator effect that can cause dilatation of smooth peripheral vessel causing blood pressure decreased (Ren, 2019). Milrinone effective for short term treatment, long-term treatment effectiveness is not confirmed yet. Milrinone mechanism (Dabbagh et al., 2017):

A) Exerting relaxation in arterial blood vessel smooth muscle

B) Inducing myocardial contractility (positive inotropic effect),

C) Improving Frank-starling curve in perioperative patient with low cardiac output (positive inotropic effect)

D) Increasing in systolic function, and diastolic relaxation.

Milrinone is indicated in perioperative patient with low cardiac output (with systolic and/or diastolic dysfunction), heart failure (including cardiogenic shock), and pulmonary hypertension. Administration of milrinone divided into loading dose and maintenance dose (Dabbagh et al., 2017):

- Loading dose administration

$25-75 \mathrm{mcg} / \mathrm{kg}$ for patient with cardiopulmonary bypass (CPB) (often given bolus during $\mathrm{CPB}$ ), and intravenous administration should be indicated in patient without cardiopulmonary bypass for 10-60 minutes. Blood pressure should be controlled.

- Maintenance dose administration 0.25- $0.75 \mathrm{mcg} / \mathrm{kg} / \mathrm{min} \quad \mathrm{IV}$, maintenance dose can be given instead of loading dose because loading dose cause initial hypotension. Treatment starts with infusion; evaluation of therapeutic plasma levels will improve after several hours of administration.

Milrinone is contraindicated in patient with hypersensitivity, obstructive 
valve lesion, decreased AV node impulse delay causing increased ventricular responds, diuretic patients (induce abnormalities in renal perfusion and electrolyte balance), and decreased ventricular filling (severe hypotension) (Dabbagh et al., 2017). Adverse effect of milrinone is commonly arterial hypotension, compared to dobutamine, milrinone induce less tachycardia with more vasodilation, arrythmias, thrombocytopenia, myocardial ischemia, but Milrinone does not increase myocardial oxygen demand (Zimmerman et al., 2019).

\section{Dobutamine}

Dobutamine has stronger beta effects (dominant in beta-1 receptor) than alpha effects. Dobutamine cause systemic vasodilation and increase inotropic state. Dobutamine will cause reduction in systemic vascular resistance with increase in heart rate and blood pressure. Compared with epinephrine or dopamine, dobutamine reduces systemic vascular resistance through direct vasodilation and decrease in sympathetic vascular tone. This mechanism will increase cardiac output without changing mean arterial pressure (MAP) (Zimmerman et al., 2019).

Dobutamine is indicated in cardiac decompensation, acute heart failure, cardiogenic shock, distributive shock, and congestive heart failure. Prescription can be given through infusion $2-20 \mathrm{mcg} / \mathrm{kg} / \mathrm{min}$ IV/IO and titrated until giving effect. Administration more than $20 \mathrm{mcg} / \mathrm{kg} / \mathrm{min}$ induce tachycardia, ventricular ectopy, and exacerbation of myocardial ischemia. Infusion must be tapered in 48-72 hour since administration given. Adverse effect of dobutamine includes ectopic heartbeats, blood pressure (BP) and heart rate elevation, hypotension, arrythmia with hypokalemia risk, and increase of myocardial demand (Dabbagh et al., 2017).

\section{Levosimendan}

Levosimendan causes cardiomyocyte more sensitive to ion intracellular calcium leading to rise of contractility. Inotropic effect in Levosimendan induces peripheral vasodilation by opening ATP-sensitive potassium channel in blood vessel. The use of Levosimendan is unessential to have renal and hepatic dose adjustment. And it has no effect on arrythmia. Loading dose of levosimendan 16-12 mcg/kg IV over 10 minutes continued by IV $0.05 \mathrm{mcg} / \mathrm{kg} / \mathrm{min}$, effect will start in 5 minutes until 10-30 minutes and effects will stay in one to twohour, infusion should be given more than 24 hours. Levosimendan side effect include headache with or without hypotension due to vasodilatory effect of the drug, risk for arrythmia is not found, no renal or hepatic dose adjustment needed, and no myocardial oxygen demand. Not enough mortality data found for pediatric patient (Dabbagh et al., 2017).

H. Pure vasodilators (arterial dilators and/or venodilator)

Pure vasodilator agents are important to be used in patients with cardiogenic shock 
secondary to left-right shunt, low cardiac output after operation, severe atrioventricular valve regurgitation, and dilated cardiomyopathy. Pure vasodilators have no inotropic activity, for example nitroglycerin, hydralazine, alprostadil, sodium nitroprusside, phentolamine mesylate.

\section{Nitroglycerin}

Administration of nitroglycerin will induce relaxation of vascular smooth muscle by stimulating intracellular cyclic guanosine monophosphate production. Nitroglycerin therapeutic dosage is $0.5-5 \mathrm{mcg} / \mathrm{kg} / \mathrm{min}$. Dosage from $0.5-2 \mathrm{mcg} / \mathrm{kg} / \mathrm{min}$ induce venodilator effect, meanwhile, 2-5 $\mathrm{mcg} / \mathrm{kg} / \mathrm{min}$ improves cardiac index, decrease pulmonary and systemic blood pressure. Hydralazine is an antihypertensive agent (Dabbagh et al., 2017).

\section{Hydralazine}

One of antihypertensive drug that lowers blood pressure with vasodilating effect in peripheral. Vasodilating effect of hydralazine occurs calcium flow blockade in vascular smooth muscle. In pediatric patient, hydralazine is used as secondary treatment of oral antihypertensive when the first-line treatment failed. Common dosage used for oral dose is $0.75 \mathrm{mg} / \mathrm{kg}$ daily $\left(25 \mathrm{mg} / \mathrm{m}^{2}\right)$ divided into 4 doses and dosage can gradually be increased up to $7.5 \mathrm{mg} / \mathrm{kg}$ daily (200 mg daily). Parenteral dosage can be administered if severe hypertension happens. Hydralazine is contraindicated in patient with mitral valvular rheumatic heart disease and CAD. It can cause pyridoxine insufficiency and peripheral neuritis, and neurological symptom (Dabbagh et al., 2017).

\begin{tabular}{|c|c|c|c|c|c|c|c|c|}
\hline Drug & $\alpha$ Receptor & $\boldsymbol{\beta}_{1}$ Receptor & $\boldsymbol{\beta}_{2}$ Receptor & Cardiac Output & Heart Rate & SVR & MAP & PVR \\
\hline Epinephrine & H & H & H & $\uparrow$ & $\uparrow$ & $\uparrow$ & $\uparrow$ & 0 \\
\hline Isoproterenol & 0 & H+ & $H+$ & $\uparrow$ & $\uparrow$ & $\downarrow$ & $\downarrow$ & 0 \\
\hline Norepinephrine & H & H & 0 & 0 & 0 & $\uparrow$ & $\uparrow$ & $\uparrow$ \\
\hline Dopamine & H & H & 0 & $\uparrow$ & $\uparrow$ & $\uparrow$ & $\uparrow$ & 0 \\
\hline Dobutamine & 0 & H+ & + & $\uparrow$ & $\uparrow$ & $\downarrow$ & $\downarrow$ & $\downarrow$ \\
\hline Milrinone & 0 & 0 & 0 & $\uparrow$ & 0 & $\downarrow$ & $\downarrow$ & $\downarrow$ \\
\hline Phenylephrine & H+ & 0 & 0 & 0 & $\downarrow$ & $\uparrow$ & $\uparrow$ & $\uparrow$ \\
\hline Vasopressin & 0 & 0 & 0 & 0 & 0 & $\uparrow$ & $\uparrow$ & 0 \\
\hline Ephedrine & + & + & + & $\uparrow$ & $\uparrow$ & $\uparrow$ & $\uparrow$ & 0 \\
\hline Levosimendan & 0 & 0 & 0 & $\uparrow$ & 0 & $\downarrow$ & $\downarrow$ & $\downarrow$ \\
\hline
\end{tabular}

Figure 3. Vasopressors and inotropes differences based on receptor and physiologic effects (Zimmerman et al., 2019). 


\section{Electrolyte balance management}

Evaluate any abnormalities in electrolyte balance because it can affect the myocardial function. For example, electrolyte that should be managed are potassium, calcium, and magnesium (Chiwane et al., 2018).

\section{Afterload management}

Reducing afterload by using vasodilator or positive pressure ventilation (PPV) is the only intervention that can improve stroke volume without increasing end-diastolic pressure. PPV helps reducing afterload greatly for left ventricle (Chiwane et al., 2018).

Selective vasodilator infusion (sodium nitroprusside, esmolol, and nicardipine) can be prescribed. For patient with severe aortic insufficiency or AV regurgitation, administration of antihypertensive drugs is able to decrease systemic vascular resistance (SVR) making stroke volume improved (Chiwane et al., 2018).

Milrinone (PDE inhibitor class III) from inotropic family can be used to reduce systemic and pulmonary afterload. Pulmonary vasodilator (inhaled nitric oxide and milrinone) can be administered for patient with right ventricular dysfunction. Prostaglandin can be used to reduce afterload in children with coarctation of the aorta
(Chiwane et al., 2018). After load can be reduced using mechanical intervention which includes intra-aortic balloon pump (IABP), extracorporeal membrane oxygenation (ECMO), ventricular assist device (VAD), and abdominal compression device. ECMO is the most common mechanical circulatory support which is used for pediatric patient. Venoatrial (VA) ECMO is the mode used in infant and children with myocardial dysfunction. Three modes of ECMO cannulation used for pediatric patient include (Cashen et al., 2018):

A. Transthoracic cannulation

B. Right internal jugular vein and right carotid artery percutaneously cannulation or through cervical incision

C. Femoral vein and femoral artery cannulation

Cannulation depends on the size of vessel, obstructed vessel, cardiac anatomy, dan surgeon preference. Peripheral cannulation through the carotid artery and internal jugular vein are commonly used for pediatric and infant patient as long as there's no contraindication. ECMO help patient in recovery, transplant, and many mechanical assists to place device, or and cardiopulmonary resuscitation. The main benefit using ECMO is that it can be rapidly deployed, and done in a child with closed chest, and provide cardiac support well (Cashen et al., 2018). 


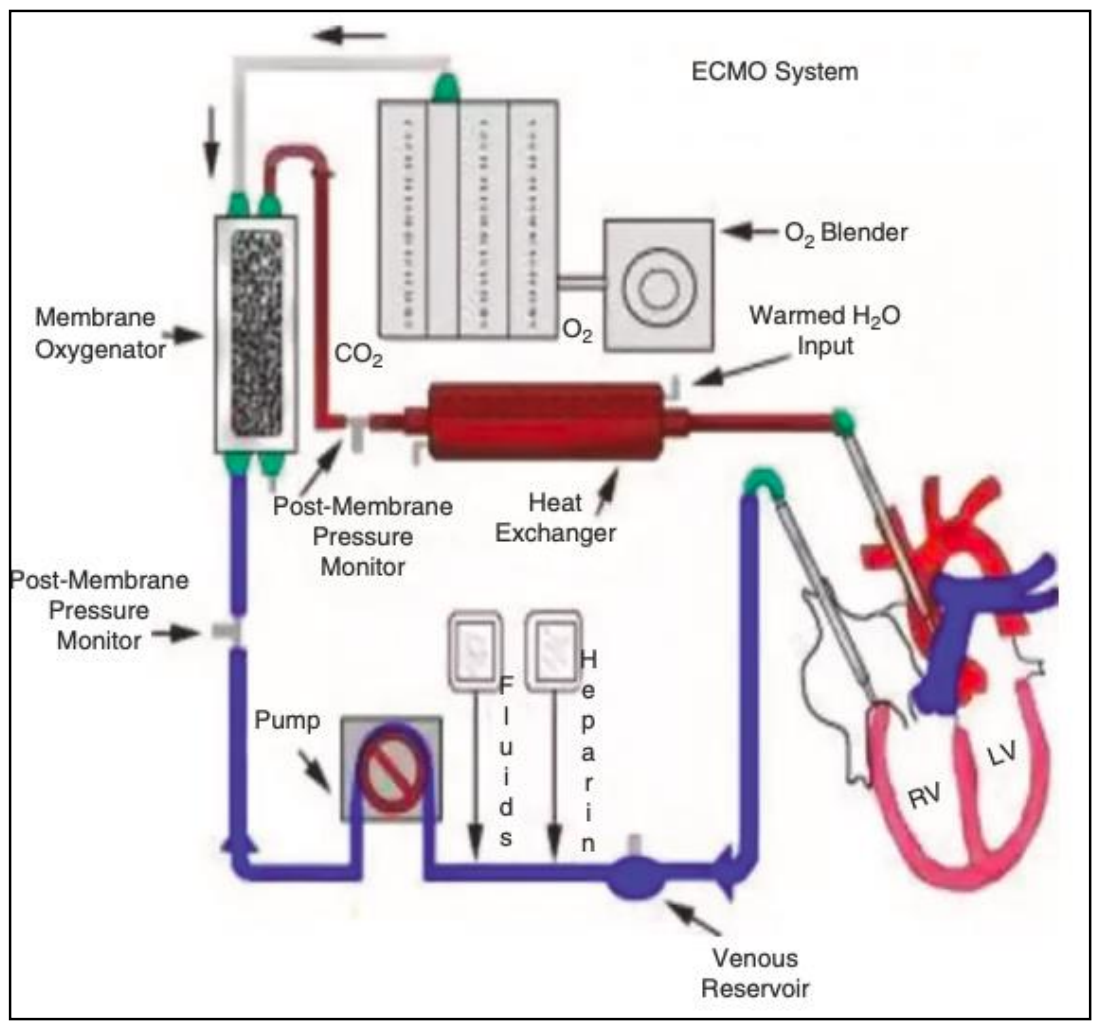

Figure 4. ECMO (Extracorporeal membrane oxygenation) Mechanism pumping blood from circulation through artificial lung back into bloodstream (Cashen et al., 2018).

\section{Atrioventricular (AV) synchronicity management}

$$
\text { By evaluating and correcting }
$$
electrolyte concentration, arrhythmias can be prevented. In some cases where arrythmia is commonly found such as acute myocarditis, cardiomyopathy, and coronary ischemia, the use of catecholaminergic inotropic agents should be avoided or use the lowest doses if it's possible. Recommended treatment for arrhythmias is pharmacotherapy, pacing, cardioversion, or defibrillation (Chiwane et al., 2018).

\section{Increasing arterial oxygen carrying capacity}

Increasing arterial oxygen carrying capacity improves not only systemic oxygen delivery but also myocardial oxygen delivery hence it can improve myocardial function. PPV (positive pressure ventilation) could increase lung's oxygenation by improving functional residual capacity. Administering packed red blood cell transfusion helps improving oxygen carrying capacity but transfusion is limited only to induce myocardial contractility or in severe blood loss. Side effect of transfusion include volume overload and increasing fluid 
viscosity which cause afterload increasing (Chiwane et al., 2018).

From the points mentioned before, it can be concluded that these principal therapies can improve oxygen delivery by improving cardiac output. When oxygen delivery improved, arterial oxygen carrying capacity and hemoglobin concentrations can be corrected (Chiwane et al., 2018).

\section{Complications}

Shock is the main cause of morbidity and mortality in PICU (Lee et al., 2017). Cardiogenic shock complication depends on adequate management and early diagnosis. severe cardiogenic shock, the body's organs do not get enough oxygen-rich blood. This can cause temporary or permanent damage to the vital organs of your body. Complication of cardiogenic shock includes cardiopulmonary arrest, dysrhythmia, renal failure, multisystem organ failure, and death (Ren, 2019).

\section{Prognosis}

The key to improve outcomes and decrease mortality in pediatric patients with cardiogenic shock is early identification, evaluation and treatment. However, despite improvements in both medical and mechanical management, morbidity and mortality remain high as compared to other forms of pediatric shock. The current estimated mortality rate is as high as 5 to 10 percent, but increases up to fivefold in the presence of comorbidities such as acute kidney or liver failure and sepsis. Early myocardial support, both with medical or mechanical support, can improve end-organ function and perfusion, and thus reduce morbidity and mortality in this patient population (Brissaud et al., 2016).

A study by Othman et al, in 2020 reviews patients' data from National Inpatient Sample from 2002-2016. The study reveals that patients with cardiogenic shock that stemmed from cardiomyopathies had higher mortality than those that had congenital heart disease. The use of ECMO had a comparable rate between the two groups, while heart transplantation and the use of ventricular assist device were significantly higher in those with cardiomyopathies (Othman et al., 2020).

\section{Summary}

Shock is an acute failure of the cardiovascular system to meet the metabolic demands of the tissues. Inadequate tissue oxygenation can lead to anaerobic metabolism and acidosis, and eventually, loss of cellular function and organ dysfunction.

Diagnosis of cardiogenic shock can be made from clinical signs and symptoms, certain tests may also be needed to define the underlying cause of the shock (myocardium functional status and other comorbid features), and to evaluate therapeutic response.

Basic treatment for any kind of shock is to optimize oxygen delivery so that it can meet the oxygen demand. Fluid resuscitation crystalloid fluid $5-10 \mathrm{~mL} / \mathrm{kg}$ 
boluses ought to be administrated. Evaluate any signs of volume overload. Diuretic is mainly used to treat systemic and pulmonary vascular congestion. Initial treatment with inotropic agents is recommended to increase myocardial contractility.

\section{REFERENCES}

1. Bankir, Lise., Bichet, Daniel G., Morgenthaler, Nils G., 2017. Vasopressin: physiology, assessment, and osmosentation. Journal of internal medicine. 282(4).

2. Brissaud, O., Botte, A., Cambonie, G., Dauger, S., Blanquat, L.S., Durand, P., Gournay, V., Guillet, E., Laux, D., Leclerc, F., Mauriat, P., Boulain, T., Kuteifan, K. 2016. Experts' recommendations for the management of cardiogenic shock in children. Ann Intensive Care. $6: 14$

3. Cashen, Katherine., Sehgal, Swati., Walters, Henry L. 2018. Cardiac emergencies in children: Pediatric Mechanical Circulatory Support. Card Emergencies Child A Pract Approach to Diagnosis Manag. Springer International Publishing. 1(16):267 - 283

4. Sarnaik AP, Ross RD, Lipshultz SE, Walters HL. 2018. Cardiac emergencies in children: A practical approach to diagnosis and management. Card Emergencies Child A Pract Approach to Diagnosis Manag. 1-394.
5. Dabbagh A, Conte AH, Lubin L. 2017. Congenital heart disease in pediatric and adult patients: Anesthetic and perioperative management. Congenital Heart Disease in Pediatric and Adult Patients: Anesthetic and Perioperative Management. 1-1025 $\mathrm{p}$

6. Hayabuchi, Y., Homma, Y., Kagami, S. 2019. A novel index equivalent to the myocardial performance index for right ventricular functional assessment in children and adolescent patients. $S c i$ Rep 9, 19975

7. Kataja, A., Harjola, V.P. 2017. Cardiogeni shock: current epidemiology and management. Continuing Cardiology Education. $3: 3$

8. Kliegman, R.M., St.Geme, J.W., Blum, N.J., Shah, S.S., Tasker, R.C., Wilson, K.M., Behrman, R.E. 2020. Nelson Textbook of Pediatric $21^{\text {st }}$ Ed. Philadelphia. Elsevier

9. Mendelson, J. 2018. Emergency Department Management of Pediatric Shock. Emerg Med Clin N Am. 
10. Migally, K., McBride, ME. 2018. Cardiogenic Shock Beyond The Neonatal Period. Elsevier. 19(4).

11. Othman, H.F., Hamzah, M., Aly, H. 2020. Outcomes of Cardiogenic Shock in Pediatric Cardiomyopathy Versus Congenital Heart Disease: Analysis of The National Inpatient Sample Database. Journal of the American College of Cardiology 75(11): 1

12. Pasman, Eric A. 2019. Shock in Pediatrics Treatment $\&$ Management. Medscape. https://emedicine.medscape.com/art icle/1833578-treatment\#d1.

[Accessed 25 August 2020].

13. Ren, X., 2020. What Is the Indication for Diuretics in The Treatment of Cardiogenic Shock? Medscape. https://emedicine.medscape.com/art icle/152191-treatment\#d11.

[Accessed 25 August 2020].

\section{ABBREVIATIONS}

ALCAPA: anomalous left coronary artery from the pulmonary artery

AV: atrioventricular

BP: blood pressure

cAMP: cyclic adenosine monophosphate

CPB: cardiopulmonary bypass

CRT: capillary refill time

ECG: electrocardiography

ECMO: extracorporeal membrane oxygenation
14. Richards, E., Lopez, M. and Maani, C., 2020. Phenylephrine. Ncbi.nlm.nih.gov. https://www.ncbi.nlm.nih.gov/book s/NBK534801. [Accessed 28 August 2020].

15. Singh, Y., Katheria, A.C., Vora, F. 2018. Advances in diagnosis and management of hemodynamic instability in neonatal shock. Front. Pediatr. 6:2

16. Stephenson, M. 2020. Understanding shock in children. British Journal of Nursing. 29: p350-352.

17. Zimmerman J, Lee JP, Cahalan M. Vasopressors and inotropes [Internet]. Second Edition. Pharmacology and Physiology for Anesthesia: Foundations and Clinical Application. Elsevier Inc.; 2018. p520-534.

e-FAST : extended focused assessment sonography for trauma

EGDT: early goal directed therapy

FAST: focused assessment sonography for trauma

HLHS: hypoplastic left heart syndrome IABP: intra-aortic balloon pump

ICT: isovolumetric contraction time IO: intraosseous

IRT: isovolumetric relaxation time IV: intravenous IVS: intact ventricular septum 
MAP: mean arterial pressure

MPI: myocardial performance index

PDA: patent ductus arteriosus

PDE: phosphodiesterase

PICU: pediatric intensive care unit $\mathrm{v}$

PPV: positive pressure ventilation

$\mathrm{SvO}_{2}$ : mixed venous oxygen saturation

SVR: systemic vascular resistance

TAPVR: total anomalous pulmonary venous

return

TGA: transposition of great aorta

TOF: tetralogy of fallot

VA: venoatrial

VAD: ventricular assist device

VET: ventricular ejection time

VSD: ventricular septum defe 
\begin{tabular}{l|l} 
Page | 61 & 01
\end{tabular} 